\title{
Pathogenesis of cardiac graft failure in children*
}

\author{
Jenny Lin, Bahig M. Shehata \\ Department of Pathology, Children's Healthcare of Atlanta, Atlanta, USA \\ Email: bshehat@emory.edu
}

Received 26 April 2012; revised 16 July 2012; accepted 5 September 2012

\begin{abstract}
For many critical cardiac conditions such as cardiomyopathy, congenital heart defects, or arrhythmogenic disorders, cardiac transplantation is often an accepted treatment with optimistic one- and five-year survival rates of $90 \%$ and $75 \%$ in the pediatric patient population, respectively. However, poor longterm survival is a cause for concern, with cardiac graft failure being the leading cause in late mortality transplant recipients. Cardiac graft failure occurs through several mechanisms. However, the most common causes include cardiac allograft vasculopathy, myocardial fibrosis, and fibrofatty changes. Risk factors exacerbate these mechanisms through rejection, immunosuppressive drug side effects, hypertension, diabetes mellitus, renal dysfunction, and viral infection to increase the chance of cardiac graft failure. Changes in expression levels of various genes offer diagnostic potential for the future of cardiac transplantation. Through a comprehensive review of how multiple factors can lead a cardiac graft into failure, we hope to contribute to the longevity of cardiac grafts and pediatric heart transplant recipients.
\end{abstract}

Keywords: Cardiac Graft Failure; Pediatric Cardiac Transplant

\section{INTRODUCTION}

For over 25 years, cardiac transplantation has been proven to be a viable treatment option for children with end-stage heart failure, attributable to cardiomyopathies, Congenital Heart Defects (CHD), and, to a lesser extent, arrhythmogenic disorders [1,2]. Although CHD is the predominant diagnosis leading to cardiac transplants in infants at $66 \%$ with cardiomyopathy accounting for $30 \%$ of cases, the percentage of cases due to cardiomyopathy increases to $52 \%$ for children ranging from 1 to 10 years of age, and transplants as a result of CHD decreases to $37 \%$ [3]. In the adolescent age group, cardiomyopathy

"Conflict of interest: The authors declare no conflict of interest. The authors alone are responsible for thecontent and writing of this article. continues this trend, reaching a percentage of $62 \%$ in adolescents, with CHD accounting for only $28 \%$ of transplants [3]. Since the first pediatric cardiac transplantation in 1967, over 8000 heart transplants in children have been performed, and with more than 400 heart transplants conducted annually today, it is necessary to reduce the risks of graft failure and the need for retransplantation $[2,4]$. Although survival rates for recipients of cardiac transplants is $90 \%$ and $75 \%$ at 1 and 5 years, respectively, long-term survival rate is not as optimistic with survival dropping to $40 \%$ at 20 years [2,5]. Thus, it is important to understand the mechanism and etiology of cardiac graft failure in order to improve the treatment and outcomes of pediatric cardiac transplant recipients.

\section{DISCUSSION}

\subsection{Mechanisms of Graft Failure}

\subsubsection{Cardiac Allograft Vasculopathy}

Cardiac Allograft Vasculopathy (CAV) is a major diagnosis of long-term morbidity and mortality in heart transplant patients and the leading cause of death 1 year posttransplantation [6]. It is a diffuse and progressive thickening of the innermost layer of small and large epicardial and intramyocardial vessels of the transplanted heart caused by fibrous hyperplasia and lipid plaques [5-7]. It occurs early after transplantation when the innate and cell-mediated immune system targets the vascular endothelium, the border that lies between the immune cells in circulation and the vessel wall, causing intimal thickening, macrophage injury, and smooth muscle hyperplasia [7]. This intimal thickening creates ischemic and myocardial injury, invoking chronic immune response [7]. Endothelium dysfunction results in reduced local Nitric Oxide (NO) bioavailability, which encourages adhesion of platelets, monocytes, and other inflammatory cells [7]. Reduced NO also increases local oxidant stress, upregulates inflammatory genes, and activates smooth muscle cell proliferation [7].

Although CAV is similar to Coronary Artery Disease (CAD) morphologically, the two conditions differ in the initial cause of disease and plaque content. While immunological mechanisms are indirectly involved in CAD, 
immunological injury acts as the initial insult in developing CAV through graft rejection, viruses, reperfusion injury, and ischemia graft time after organ restabilization [7]. Furthermore, although plaque in both CAV and CAD contain foam cells and dead lymphocytes, a major feature of plaque content in CAV includes a greater calcium deposition [7].

Diastolic dysfunction and restrictive ventricular hemodynamics have been known to occur with CAV [5]. CAV in the denervated heart can develop rapidly without symptoms of angina, resulting in unanticipated sudden death, congestive heart failure, or arrhythmias [6]. The incidence of CAV in pediatric patients is $7 \%-11 \%$ [3].

\subsubsection{Myocardial Fibrosis}

In most cardiac conditions, fibrosis occurs as scarring in structural remodeling of the myocardium through aggregation of fibroblasts and extracellular matrix (ECM) proteins $[8,9]$. Fibrosis can develop in two forms. Reactive interstitial fibrosis derives from areas around the microvasculature and spreads throughout the myocardium to maintain the pressure generating power of the heart without loss of cardiomyocytes [8]. This often progresses into replacement fibrosis, which occurs throughout the myocardium and is characterized by cardiomyocyte hypertrophy and necrosis and decrease in cardiomyocyte mass [8]. Activated fibroblasts and the excessive ECM are responsible for this cardiomyocyte hypertrophy through paracrine signaling pathways [8]. Traditional views claim that activated fibroblasts in replacement fibrosis are derived from resident cardiac fibroblasts influenced by circulating molecules, whereas studies show that proliferating immature fibroblasts during reactive interstitial fibrosis are situated in the blood vessels and possibly from other cellular sources [8].

The widespread deposition of fibrous tissue composed of type I fibrillar collagen protein causes many unwanted consequences on the transplanted heart [9]. As the contractility of the heart worsens, the heart becomes unable to properly contract and provide an appropriate amount of blood and nutrients into the systemic circulation to the tissues of the body [9]. Furthermore, the consequential stiffness of the heart causes diastolic dysfunction by compromising its filling capacity during diastole and elevates atrial and ventricular filling pressures, displacing an insufficient amount of blood during contraction [8,9]. Since the ECM normally separates the atria and ventricles for proper contraction, the accumulation of ECM cripples the mechano-electric coupling between cardiomyocyte layers, causing increased risk of arrhythmias and abnormal cardiac function [8].

Fibroblasts encompass a wide variety of cells differentiated from the mesoderm that release a range of matrix proteins and biochemical intermediates, such as growth factors and proteases [8]. Due to their pleiomorphic nature, their expression and contractile proteins are dependent on their local microenvironment [8]. For instance, myofibroblasts and fibroblasts both produce actin and myosin proteins but at unequal amounts [8]. Although increased ECM is a main trait of fibroblasts, fibroblasts are normally identified morphologically as flat spindle shaped cells with numerous processes originating from the cell body [8]. Fibrosis in cardiac allografts is identified by increased myocardial collagen and interstitial fibrosis [5].

\subsubsection{Fibrofatty Changes}

Right ventricular fibrofatty infiltration of the myocardium was recently described by Sacks and colleagues in 2010 as another possiblecause of heart graft failure [2]. Similar to Arrhythmogenic Right Ventricular Dysplasia (ARVD), it is defined by myocyte deterioration and fibrofatty permeation [2]. The histology of fibrofatty changes includes a thinned myocardium of the right ventricle with invasion of fat, fibrotic, and myocyte vacuoles [2]. It is commonly found in the arrhythmogenic triangle of the subtricuspid area, apex, and infundibulum [2]. Since these failed cardiac grafts show no fibrofatty morphology at the time of transplantation, acquired fibrofatty changes in the graft are thought to be due to inflammation, rejection, infection, or drug therapy side effects depending on the time of rejection [2]. The extent of the fibro fatty invasion into the right ventricle has been shown to be associated with significantly reduced cardiac graft life [2]. More extensive research is needed on this entity to determine its significance as a contributor to transplanted graft failure.

\subsection{Risk Factors}

\subsubsection{Rejection}

The first year post-transplantation is characterized by varying degrees of immunological responses. The first 3 months consist of a period of heightened rejection risk which is followed by a lower risk in the next 3 months [10]. This decline in graft rejection is interrupted by either a change in immunosuppressive drug dosing or immune system activation [10]. Elevated levels of antihuman leukocyte antigen (HLA) antibodies have been correlated with hyperacute rejection, humoral rejection, and increased acute cellular rejection in heart transplantation, and this increased rejection has been found to be associated with poorer survival in early and late allograft failure $[1,11]$. Presensitization is commonly more frequent in patients receiving transplants for CHD than cardiomyopathy, which is likely related to exposure to different blood products in open-heart surgeries, blood transfusions, and Ventricular Assist Device (VAD) therapy $[1,4,11]$. De novo anti-HLA antibodies have been shown 
to be associated with a higher rate of chronic graft rejecttion [11].

The presence of ELISA-detected anti-HLA antibodies has been shown to be specifically correlated with the progression of CAV [11]. The polymorphic nature of HLA causes frequent donor-recipient mismatching [7]. Myocyte damage from ischemic injury or an acute rejection episode can perpetuate a chronic immune response through the expression of Stress-Related Proteins (SRP), such as Heat Shock Proteins (HSP), which can result in T-cell activation and primary and memory B-cell responses of anti-HLA antibodies, inducing myocyte apoptosis and necrosis [7].

Non-HLA antibodies may also be associated with chronic graft failure, such as anti-major histocompatibility complex class I related chain A (MICA) antibodies [7]. MICA antibodies are stress-inducible activating ligands for NK lymphocytes and T-cells and are upregulated in endomyocardial tissue [7]. Moreover, cytotoxic IgM antibodies have been linked to acute rejection when myocardial damage leads to C4d deposition in cardiac capillaries [7]. Microvascular damage from HLA and non-HLA antibodies may exacerbate coronary occlusion and myocardial ischemia, causing hyperactivity of cytokines, chemokines, chemoreceptors, and adhesion molecules, contributing to immune cell chemotaxis, diapedesis, and, consequently, CAV intima thickening [7].

\subsubsection{Immunosuppressive Side Effects}

Immunosuppressive drugs are commonly used after transplantation to inhibit rejection of the newly-grafted heart. These drugs include calcineurin inhibitors (CNI), or immunophilin-binding drugs, that block lymphocyte signal transduction, antiproliferative agents that block de novo nucleotide synthesis, and cytolytic biological agents [3]. Immunophilin-binding drugs include cyclosporine A (CSA), tacrolimus (Tac), and sirolimus, and examples of antiproliferative agents include azathioprine and mycophenolate mofetile [3]. Cytolytic agents can be either polyclonal drugs that are used in induction therapy against $\mathrm{T}$ cells and thymocytes or monoclonal drugs that are utilized against hemodynamically significant rejection [3].

CNIs of cyclosporine A and tacrolimus are the main immunosuppressive agents administered in the first year of heart transplantation to $37 \%$ and $57 \%$ of recipients, respectively [7]. Renal dysfunction has been linked to long-term use of CNIs, and alternatives such as sirolimus are used because of their lower nephrotoxicity and reduction in CAV development [1]. Hypertension is another side effect of CNIs through activation of the renin-angiotensin-aldosterone system and sympathetic nervous system, causing vasoconstriction and hypertension in $35 \%-65 \%$ of pediatric heart transplants [12]. In fact, endomyocardial biopsies of patients administered CSA demonstrate interstitial edema and fibrosis [7]. Coronary arteritis and calcification has also been reported with patients prescribed Tac [7]. These CNI side effects may further contribute to CAV, myocardial fibrosis, and, consequently, graft failure.

Corticosteroids, another common drug given to transplant patients, can cause hypertension through fluid retention that may contribute to CAV [7]. Another side effect of CNIs and corticosteroids is diabetes mellitus, discussed below.

\subsubsection{Renal Dysfunction}

By 10 years after transplantation, renal impairment is found in up to $40 \%$ of patients, and severe renal dysfunction is found in 14\% [7]. As mentioned previously, renal function is worsened by CNI side effects. Volume overload and hypertension with left ventricular hypertrophy and anemia are associated symptoms in chronic renal dysfunction and can all affect proper cardiac function [7]. Besides hemodynamic perturbation, uremia has been shown to increase interstitial fibrosis and microvascular changes [7]. Irregular handling of calcium by myocytes in response to uremia leads to a failure of relaxation and increase in diastolic dysfunction [7].

\subsubsection{Diabetes Mellitus}

Steroids given after transplantation increase resistance to peripheral and hepatic insulin, and CNIs may disrupt insulin release by toxically affecting pancreatic beta cells, disrupting glucose tolerance [12]. Thus, diabetes mellitus is a common occurrence in $37 \%$ of transplant patients by 10 years [7]. It is known to worsen CAV by contributing to atherosclerotic disease of coronary vessels [7]. Furthermore, reactive oxygen species produced in diabetes mellitus may lead to myocyte damage that can invoke the immune response and consequently cause CAV and fibrotic structural remodeling [7].

\subsubsection{Hypertension}

As mentioned previously, hypertension is a side effect of CNIs in immunosuppressant therapy. Ten years posttransplantation, 99\% of cardiac transplant recipients is hypertensive [7]. Heightened long-term pressure on the graft causes left ventricular hypertrophy, collagen formation, and fibroblast stimulation [7]. This restructuring increases the likelihood of cardiac graft failure through CAV and fibrosis. This results in stiffness of the left ventricle and diastolic dysfunction [7]. Prolonged hypertension may lead to atrial fibrillation, causing loss of a trial systole and abnormal ventricular rates [7].

\subsubsection{Viral Infection}

Cardiac transplant patients are prone to viral infection of the grafted heart, which has similar pathology to viral 
myocarditis consisting of inflammatory infiltrate, myocyte necrosis, edema, and fibrosis [13]. These symptoms not surprisingly can contribute to the development of CAV and fibrosis. In fact, viral genome has been found in cardiac grafts and has been associated with graft loss and rejection [13]. The viruses known to infect cardiac transplant patients include parvovirus B19, adenovirus, Epstein-Barr Virus (EBV), and cytomegalovirus (CMV) $[7,13]$.

CMV has been identified in the causative factors in CAV and has been linked to coronary endothelial dysfunction and the migration of inflammatory immune cells into vascular tissue [7]. Ganciclovir and anti-CMV immunoglobulin decrease the development of transplant coronary artery disease [13]. Intravenous immunoglobulin can also treat viral myocarditis and may improve graft survival against other infectious agents [13].

EBV infection after transplantation precedes development of Post-Transplant Lymphoproliferative Disorder (PTLD), a lymphoid tumor associated with immunosuppressed patients [3]. Clinical diagnosis ranges from a nonspecific viral syndrome, to mononucleosis, and to malignant lymphoma [3].

Other organisms besides viruses may also contribute to the development of graft failure. The parasitic protozoa Toxoplasma gondii, although not a virus, also encourages the cell-mediated immune response and the development of acute CAV [7].

\subsection{Genetics}

Presently, the genetic etiology of graft failure and CAV is not well known. Many single-gene mutations and polymorphisms have been proposed in multiple studies but an overwhelming consensus has not yet been achieved. This may be due to the discrepancies among experimental protocols and patients. For instance, studies have differed in their definition and grading of acute rejection, and subject populations have varied in ages, ethnicities, and immunosuppressive therapies [14].

Genetic mutations may act through various mechanisms during heart graft loss. A mutation may alter protein structure and affect its responsiveness to alloimmune interactions, pharmacology of immunosuppressive drugs, or organ systems that interact with the cardiac graft [10]. Genes involved in these mechanisms include genes encoding for products in alloimmune functions (cytokines, chemokines, cell adhesion molecules, growth factors), the renin-angiotensin-aldosterone system, or the transforming growth factor-beta superfamily $[10,14]$.

Genes involved in immune responses have been more frequently studied with CAV and graft failure. Cytokines and growth factors, for instance, are a focal point of transplant-related rejection due to their role in the regulation of lymphocyte and endothelial cell functions [14].
Cytokines are responsible for directing leukocytes to the graft location, lymphocyte differentiation and maturation, and altering endothelial cells in the vascular system of the graft [14]. The IFN- $\gamma$ gene activates the cellular and humoral immune responses and has been shown to be significantly upregulated in patients with higher risk of developing CAV [14]. Platelet-Derived Growth Factor (PDGF) directs smooth muscle cell differentiation and proliferation at sites of injury, which is likely to contribute to intimal thickening in CAV [14]. Tambur et al. in 2006 demonstrated that the AA phenotype in position -286 of PDGF-B correlated with an increased risk of CAV, whereas the GG phenotype at -286 and the CC phenotype at position -1135 correlated with a lower risk of CAV [14]. Similarly, Vascular Endothelial Growth Factor (VEGF), a growth factor involved in the differentiation of vascular progenitor cells into endothelial cells, showed an increase in CAV risk with patients of the GG phenotype at -1154 and a decrease in risk in those who typed as AC at -2578 [14].

TNF- $\alpha$, a pro-inflammatory cytokine released by macrophages, is expressed during inflammation and activates adhesion molecules and expression of the Major Histocompatibility Complex (MHC) [15]. It is also involved in activation of endothelial cells, vasodilation, vascular permeability, and myocardial deterioration [15]. Thus, it is commonly upregulated during intimal formation and plaques in coronary vessels of patients exhibiting angina and myocardial infarction [15]. TNF- $\alpha$ and TNF- $\beta$ are genes located in a polymorphic region adjacent to the MHC III region of chromosome 6 , and patients homozygous for TNFA2 and TNFB2 have had increased incidence of CAV and a higher mortality rate post-transplantation [15].

More recently, Mehra et al. have studied genes in the peripheral blood that may possibly be associated with CAV. The genes IL1R2, FLT3, ITGAM, for example, were found to be downregulated in patients with rejection grades 3A or higher in the first 12 weeks [10]. These genes are involved in corticosteroid responsiveness and can reasonably induce graft rejection in the post-transplant period during immunosuppressive therapy when a high level of corticosteroids is administered for organ recovery. IL1R2 and FLT3 are specifically involved in activation of hematopoietic precursors in the bone marrow [10]. Other genes activated by corticosteroids include IL1R1, TSC22D3, FKBP5, THBS1, and CD163 [10]. PDCD1 is a cell-membrane receptor in signaling pathways of T-cell activation and was found to be upregulated and correlated with future rejection [10]. Other genes activated by T-cell activation or cytokines includes ADA, GZMA, TRBC1, FLT3LG, NFKB1, and TNF [10]. Upregulation of any of these genes may signify the cellmediated immunological response rejecting the newly 
grafted heart.

Endomyocardial biopsy (EMB) has been the accepted follow-up procedure of cardiac grafts especially in the first year following transplantation. In fact, patients typically experience 13 to 15 cardiac biopsies in the first year alone [10]. Due to the ability of these genes in informing physicians of the immunological status of patients with respect to cardiac grafts, these genes show potential in predicting graft loss and failure in a noninvasive method that can be used as an alternative or supplement to EMBs.

\section{CONCLUSION}

Cardiac graft failure occurs with few presented symptoms in the transplant recipient, often resulting in unanticipated sudden cardiac death. The most common mechanisms from which the cardiac graft is compromised are through CAV andmyocardial fibrosis. Once initially compromised, a scaffold of reactions can perpetuate further loss of the graft through immunological responses, abnormal cardiac function, or cancer development. Genes associated during graft loss can be used as predictors of future cardiac graft failure and diagnostic tests to individualize immunosuppressive treatments of patients posttransplantation. With this comprehensive approach in analysis of cardiac graft failure, we hope to improve the understanding of cardiac grafts andthe outcomes of pediatric transplant recipients.

\section{REFERENCES}

[1] Mahle, W.T. (2008) Cardiac retransplantation in children. Pediatric Transplantation, 12, 274-280. doi:10.1111/j.1399-3046.2007.00854.x

[2] Sacks, J.H., Mahle, W., Abramowsky, C.R., Steelman, C.K., Kanter, K.R., Vincent, R.N., Berg, A. and Shehata B. (2010) Fibrofatty changes in failed pediatric cardiac allografts. Pediatric and Developmental Pathology, 14, 194-197.

[3] Milanesi, O., Cerutti, A., Biffanti, R., Salvadori, S., Gambino, A. and Stellin, G. (2007) Heart transplantation in pediatric age. Journal of Cardiovascular Medicine, 8, 6771. doi:10.2459/01.JCM.0000247439.89946.20

[4] Huebler, M., Schubert, S., Lehmkuhl, H.B., Weng, Y., Miera, O., Alexi-Meskishvili, V., Berger, F. and Hetzer, R. (2011) Pediatric heart transplantation: 23-year singlecenter experience. European Journal Cardio-Thoracic Surgery, 39, e83-e89. doi:10.1016/j.ejcts.2010.12.067

[5] Perens, G., Li, F., Meier, S., Kaur, R., Alejos, J.C. and Fishbein, M. (2009) Clinico-pathologic findings in end- stage pediatric heart transplant grafts. Pediatric Transplantation, 13, 887-891. doi:10.1111/j.1399-3046.2008.01087.x

[6] Hollenberg, S.M., Klein, L.W., Parrillo, J.E., Scherer, M., Burns, D., Tamburro, P., Oberoi, M., Johnson, M.R. and Costanzo, M.R. (2001) Coronary endothelial dysfunction after heart transplantation predicts allograft vasculopathy and cardiac death. Circulation, 104, 3091-3096. doi:10.1161/hc5001.100796

[7] Khan, U.A., Williams, S.G., Fildes, J.E. and Shaw, S.M. (2009) The pathophysiology of chronic graft failure in the cardiac transplant patient. American Journal of Transplantation, 9, 2211-2216. doi:10.1111/j.1600-6143.2009.02807.x

[8] Krenning, G., Zeisberg, E.M. and Kalluri R. (2010) The origin of fibroblasts and mechanism of cardiac fibrosis. Journal of Cellular Physiology, 225, 631-637. doi:10.1002/jcp.22322

[9] Gandhi, M.S., Kamalov, G., Shahbaz, A.U., Bhattacharya, S.K., Ahokas, R.A., Sun, Y., Gerling, I.C. and Weber, K.T. (2011) Cellular and molecular pathways to myocardial necrosis and replacement fibrosis. Heart Failure Reviews, 16, 23-34. doi:10.1007/s10741-010-9169-3

[10] Mehra, M.R., Uber, P.A. and Benitez, R.M. (2010) Genebased bio-signature patterns and cardiac allograft rejection. Heart Failure Clinics, 6, 87-92. doi:10.1016/j.hfc.2009.08.010

[11] Di Filippo, S., Girnita, A., Webber, S.A., Tsao, S., Boyle, G.J., Miller, S.A., Gandhi, S.K. and Zeevi, A. (2005) Impact of ELISA-detected anti-HLA antibodies on pediatric cardiac allograft outcome. Human Immunology, 66, 513518. doi:10.1016/j.humimm.2004.12.008

[12] LaRosa, C., Baluarte, H.J. and Meyers, K.E. (2011) Outcomes in pediatric solid-organ transplantation. Pediatric Transplantation, 15, 128-141. doi:10.1111/j.1399-3046.2010.01434.x

[13] Moulik, M., Breinholt, J.P., Dreyer, W.J., et al. (2010) Viral endomyocardial infection is an independent predictor and potentially treatable risk factor for graft loss and coronary vasculopathy in pediatric cardiac transplant recipients. Journal of the American College of Cardiology, 56, 582-592. doi:10.1016/j.jacc.2010.02.060

[14] Tambur, A.R., Pamboukian, S., Costanzo, M.R. and Heroux, A. (2006) Genetic polymorphism in platelet-derived growth factor and vascular endothelial growth factor are significantly associated with cardiac allograft vasculopathy. The Journal of Heart and Lung Transplantation, 25, 690-698. doi:10.1016/j.healun.2006.02.006

[15] Ternstrom, L., Jeppsson, A., Ricksten, A. and Nilsson, F. (2005) Tumor necrosis factor gene polymorphism and cardiac allograft vasculopathy. The Journal of Heart and Lung Transplantation, 24, 433-438. doi:10.1016/j.healun.2004.02.019 